\title{
Controllo dei composti organici volatici mediante fotobioreattore algale-batterico
}

\author{
Carmine Antonio Budetti ${ }^{1}$ \\ ${ }^{1}$ Affiliation not available
}

\begin{abstract}
Oggigiorno si è continuamente esposti a sostanze potenzialmente tossiche per la salute, come nel caso dei composti organici volatili (COV); in questo lavoro sono state individuate le origini di queste sostanze, i possibili danni derivati dall'esposizione con esse, oltre ad alcune tipologie di trattamento per il loro abbattimento. Ci si è soprattutto soffermati sul confronto tra una tecnologia biologica convenzionale, con filtro biotrickling (BTF) ed una innovativa, rappresentata da un fotobioreattore tubolare algale-batterico (TPBR), prendendo in esame l'abbattimento del toluene, uno dei COV più diffusi, appartenente alla famiglia dei BTEX. Il TPBR ha mostrato efficienze medie di rimozione del toluene vicine a quelle del BTF ma è risultato più stabile, a differenza di quest'ultimo che risulta influenzato dalla presenza di metaboliti secondari.
\end{abstract}

\section{Composti organici volatili: natura, rischi e strategie di controllo}

I composti organici volatili (COV o VOC) sono tra le sostanze più nocive presenti al mondo e nel nostro caso poniamo l'attenzione ad un sottoinsieme, quello dei BTEX, composto da benzene, toluene, etilbenzene e tre isomeri dello xilene (p-xilene, m-xilene e o-xilene) ${ }^{1}$. Possiamo trovare i COV sia in natura, in zone umide, foreste, oceani, vulcani ${ }^{2}$, ma soprattutto creati da attività antropiche, come le industrie manifatturiere, petrolchimiche (Fig. 1), emissioni dei veicoli ${ }^{2}$, vernici, 
solventi, rifiuti e impianti di trattamento di acque reflue ${ }^{3}$. Sono noti per la contaminazione del suolo, dell'aria e dell'acqua e nel settore chimico sono utilizzati come composti di partenza per ottenere plastiche, resine, detergenti, pesticidi e vernici ${ }^{4}$. Degli studi iniziali, collegati all'uso dei pesticidi, hanno individuato il miele come buon indicatore della qualità ambientale e sentinella per il biomonitoraggio ${ }^{5}$.

L'inquinamento di BTEX in acqua è dovuto invece a scarichi di acque reflue da processi industriali, rilascio di prodotti di serbatoi di stoccaggio e uso di solventi ${ }^{4}$; studi recenti hanno dimostrato per la prima volta la presenza di questi composti in ambiente acquatico su mozziconi di sigarette ${ }^{6}$. Altre emissioni di COV le individuiamo all'interno degli edifici, anche con un livello 2-10 volte superiore rispetto all'esterno, a causa di prodotti per ufficio, materiali sintetici, detergenti, deodoranti per ambienti, prodotti per la manutenzione ${ }^{7}$. Si può limitare l'emissione indoor scegliendo materiali a bassa emissione per l'arredamento e per la costruzione, oltre ad una maggiore frequenza di ricircolo dell'aria ${ }^{2}$ Questi composti arrivano all'uomo tramite ingestione, inalazione ed esposizione cutanea ${ }^{1}$; possono provocare danni come: debolezza, affaticamento, perdita di appetito, confusione e nausea ${ }^{6}$, irritazione della pelle, degli occhi, delle mucose carenza del sistema nervoso ${ }^{4}$, fino ad insorgenza di anemia e leucemia ${ }^{1}$.

Altra problematica che colpisce la popolazione è rappresentata dall'emissione di odori, tanto da essere considerati la principale causa di fastidio per via dell' esposizione ad impianti di ingegneria sanitaria ${ }^{8}$; gli impatti non sono solo legati alla salute, come disturbo del sonno, emicrania e perdita di appetito ${ }^{9}$ ma sono anche legati all'economia del turismo e sui costi del territorio ${ }^{10}$. Tra le tecniche per rilevare gli odori ricordiamo innanzitutto il sistema olfattivo umano, che è quella più accurata e che ha spinto la comunità scientifica a esplorare nuovi metodi modellati in base al suo meccanismo, sfruttando ad esempio le reti neurali artificiali (ANN) ${ }^{11}$; altre tecniche sono legate a nuove generazioni di IOMS (sistemi strumentali di monitoraggio degli odori) che riescono a controllare e validare in continuo i dati acquisiti mediante un sistema di auto calibrazione ${ }^{12}$.

Modelli classici 
sono quelli di dispersione, utili sia per il controllo che per la gestione degli odori ${ }^{13}$; un caso studio analizzato con questi modelli è quello di Borgo Montello, dove è presente una discarica di RSU e si è dimostrato che i livelli di esposizione più elevati agli odori sono stati registrati di notte, quindi in un arco temporale in cui non vi è movimentazione di rifiuti ${ }^{14}$. Studi legati all' analisi olfattometrica inoltre hanno mostrato che la concentrazione di odori in campioni di aria proveniente da impianti di ingegneria sanitaria diminuisce nel tempo trascorso dalla fase di campionamento ${ }^{15}$.

Le tecnologie usate per l'abbattimento dei BTEX e del toluene in particolare possono essere suddivise in due grandi famiglie:

- Tecnologie di trattamento chimico-fisico: usano delle piattaforme, ma le applicazioni per l'abbattimento dei gas di scarico carichi di toluene, con portate elevate e concentrazioni basse di COV non sono fattibili a livello economico.

- Processi biologici: alternative a basso costo, di cui ricordiamo i Filtri Biotrickling (BTF) che riducono costi operativi e presentano migliore stabilità del processo ma incontrano problemi in caso di concentrazione elevata di COV, che porta una crescita eccessiva di biomassa e ad una limitazione dell'ossigeno disponibile. Un'alternativa è data dai Fotobioreattori algali-batterici (TPBR) che sfruttano la sinergia tra microalghe e batteri e prevengono la limitazione di $\mathrm{O}_{2}$ oltre alla crescita di biomassa ${ }^{16}$.

Altri studi nell'ambito biologico sono stati effettuati sui processi di ossidazione avanzata (AOP) che permettono elevate capacità di eliminazione dei composti volatili, facendo però attenzione a controllare le emissioni di sottoprodotti ${ }^{17}$; uno dei processi AOP è l'ultrasonicazione in cui la degradazione dei gas $\mathrm{COV}$ avviene mediante ultrasuoni, con il toluene come composto rappresentativo; i risultati migliorano considerando influenza del ricircolo dell' acqua con aggiunta di Ozono ${ }^{3}$; altra tecnica studiata è stata quella di un bio-scrubber accoppiato con ozonizzazione che non solo ha un'elevata efficienza di rimozione del toluene ma previene condizioni inibenti per l'attività di 
microrganismi ${ }^{18}$

Il processo ad ultrasuoni ha mo-

strato risultati interessanti anche in combinazione con metodi di disinfezione tradizionali, rimuovendo con più facilità alcune specie di batteri e riducendo significativamente i tempi di reazione ${ }^{19}$. Altro processo biologico è quello di biodegradazione con il Bacillus amyloliquefaciens W1, un batterio capace di degradare tutti i composti della famiglia BTEX, col vantaggio di svilupparsi in coltivazione semplice e quindi permettere la produzione su larga scala ${ }^{1}$.

Una tecnologia innovativa è quella della distillazione a membrana (MD) che riduce il consumo di energia primaria sfruttando quella geotermica nelle acque reflue ma lo svantaggio è legato alle incrostazioni della membrana, che riducono efficienza energetica ed è per questo che si cerca di combinarla con adeguato pretrattamento ${ }^{20}$.

I fotobioreattori a membrana che vedremo in questo studio, anche se sotto la configurazione MPBR sono stati studiati anche per la cattura di $\mathrm{CO} 2$ e la produzione di biomassa, mostrando velocità di raccolta efficiente ma anche la riduzione di nitrati e della torbidità nel permeato ${ }^{21}$; altre applicazioni dei bioreattori a membrana MBR possono essere sviluppate in collaborazione con i nanomateriali ma il problema principale è legato ai costi su larga scala ${ }^{22}$; le prestazioni delle membrane sono vincolate anche alle dimensioni dei pori della maglia e ai tipi di materiali di supporto, come quelli polimerici che sono ampiamente utilizzati per la loro disponibilità e il basso costo ${ }^{23}$.

In relazione all' acqua e ai corpi idrici inquinati vediamo dei metodi di assorbimento su carbone attivo, processi di degradazione e soprattutto, a livello innovativo, l'adsorbimento di inquinanti disciolti in acqua per mezzo di fibre naturali, con il vantaggio di non produrre prodotti secondari e di poterle riutilizzare ${ }^{4}$. Per le emissioni indoor invece è stata studiata una tecnologia al plasma termico, per valori bassi di toluene, che però sviluppa prodotti come Ozono e NOx ${ }^{7}$. 


\section{Caso studio}

Nel nostro lavoro abbiamo studiato l'abbattimento del toluene tramite due tecnologie diverse: attraverso un filtro biotrickling convenzionale (BTF) e con un innovativo fotobioreattore tubolare algale-batterico (TPBR). Per entrambi i reattori sono stati utilizzati, come inoculo batterico, i fanghi attivi dell'impianto di trattamento delle acque reflue di Valladolid.

Il toluene è stato acquistato a Barcellona e ha una purezza del 99,8\%.

Il BTF è costituito da una colonna cilindrica rivestita in PVC, con diametro interno di 0,08 $\mathrm{m}$ e altezza di 0,79 m; essa è imballata con anelli di plastica e presenta un volume di lavoro di $4 \mathrm{~L}$. Il sale minerale (MSM) utilizzato per le operazioni viene continuamente ricircolato tramite una pompa peristaltica, da un serbatoio esterno di 1,2 L agitato a $400 \mathrm{rpm}$; il toluene viene emesso dal fondo del reattore (Fig. 2).

Il TPBR, da 45,6 L è costituito da tubi con diametro interno di $5 \mathrm{~cm}$ e lunghezza totale di 20 m; è interconnesso ad una colonna verticale da $2 \mathrm{~L}$ e ad una camera di miscelazione di $70 \mathrm{~L}$. Sono presenti dei LED ad emissione di luce bianca, disposti verticalmente su entrambi i lati del TPBR. L'emissione del toluene avviene tramite un metallo diffusore nella parte inferiore della colonna di assorbimento verticale. I sistemi hanno operato per 90 giorni, testando quattro diverse condizioni di funzionamento, aumentando sia il carico in ingresso in modo graduale ma anche la velocità di rinnovo di MSM. Le concentrazioni in ingresso e in uscita di toluene, $\mathrm{CO}_{2}, \mathrm{~N}_{2}$ e $\mathrm{O}_{2}$ nella fase gas, $\mathrm{pH}$ e ossigeno disciolto (DO) nel brodo di coltivazione sono stati analizzati ogni giorno; i campioni liquidi utilizzati per determinare la concentrazione di carbonio organico totale (TOC) e azoto totale $(\mathrm{TN})$, nitriti e nitrati sono stati presi tre volte a settima- 
na.

Al termine di ogni fase operativa sono state caratterizzate le popolazioni di microalghe e/o batteri, analizzando campioni di inoculo e brodo per il TPBR e dell'inoculo e del biofilm nel BTF; sempre al termine di ogni fase con un test di trasferimento di massa si è andati a chiarire se le prestazioni dei bioreattori fossero limitate dal trasferimento di toluene dal gas alla fase liquida, o per via di attività microbica. Alla fine dell'esperimento è stato eseguito un test di robustezza per i reattori.

All'avviamento di entrambi i bioreattori c'è stata un'efficienza di rimozione iniziale del $30 \%$ nel caso del TPBR per via della maggiore capacità di assorbimento del brodo di coltivazione; durante la prima fase i due sistemi hanno avuto prestazioni comparabili ma la presenza di alghe e batteri nel TPBR ha provocato un aumento di DO e il reattore ha mostrato concentrazioni di $\mathrm{CO}_{2}$ in uscita inferiori rispetto al BTF; quest'ultimo è stato più sensibile alla presenza di metaboliti secondari, indotti dall' aumento di concentrazione di toluene o ad una presenza limitata di $\mathrm{O}_{2}$, in fase acquosa. L'accumulo di questi metaboliti può portare instabilità ed infatti il TPBR si è dimostrato più stabile e più adattabile alle fluttuazioni del carico in ingresso, sia per una quantità maggiore di liquido coinvolto, sia per la presenza maggiore di DO nel brodo, oltre che per il consumo di $\mathrm{CO}_{2}$ da parte delle microalghe; i test di trasferimento di massa hanno sottolineato che entrambi i processi erano limitati dal trasferimento della massa di toluene dalla fase gassosa al biofilm o alla fase liquida ${ }^{16}$.

\section{Conclusioni}

Il nostro studio ci ha permesso di confrontare due tecnologie per l'abbattimento del toluene e abbiamo notato che le efficienze di rimozione sono simili tra loro e rispettivamente $88 \pm 4 \%$ per il TPBR e $86 \pm 9 \%$ per il BTF.

Le prestazioni del TPBR sono leggermente migliori ma sono soprattutto più stabili per via del- 
le alte concentrazioni di $\mathrm{DO}$ e valori più elevati di $\mathrm{pH}$; altro vantaggio è stata la riduzione di emissioni di $\mathrm{CO}_{2}$. Il BTF ha sofferto di prestazioni più instabili per via dell'accumulo di metaboliti secondari e di acidificazione del mezzo di ricircolo che va ad inibire l'attività microbica.

Possiamo in conclusione affermare che la cooperazione tra microalghe e batteri è una soluzione efficace per la rimozione del toluene e può rappresentare una sfida per il futuro.

\section{References}

1.A.Wongbunmak, Khiawjan, S., Suphantharika, M. \& Pongtharangkul, T. BTEX biodegradation by Bacillus amyloliquefaciens subsp. plantarum W1 and its proposed BTEX biodegradation pathways. Scientific Reports 10, (2020).

2.Bouchaala. VOLATILE ORGANIC COMPOUNDS REMOVAL METHODS: A REVIEW. American Journal of Biochemistry and Biotechnology 8, 220-229 (2012).

3.Comia, J. et al.. Degradation of Gaseous VOCs by Ultrasonication: Effect of Water Recirculation and Ozone Addition. in Frontiers in Water-Energy-Nexus-Nature-Based Solutions Advanced Technologies and Best Practices for Environmental Sustainability 333-336 (Springer International Publishing, 2019). doi:10.1007/978-3-030-13068-8 3 .

4.Tursi, A., Chidichimo, F., Bagetta, R. \& Beneduci, A. BTX Removal from Open Aqueous Systems by Modified Cellulose Fibers and Evaluation of Competitive Evaporation Kinetics. Water 12, 3154 (2020).

5.Alam, J. A., Fajloun, Z., Chabni, A. \& Millet, M. The use of honey as environmental biomonitor of pesticides contamination in northern Lebanon. Euro-Mediterranean Journal for Environmental Integration 2, (2017). 
6.Dobaradaran, S., Schmidt, T. C., Kaziur-Cegla, W. \& Jochmann, M. A. BTEX compounds leachates from cigarette butts into water environment: A primary study. Environmental Pollution 269, 116185 (2021).

7.Durme, J. V., Dewulf, J., Sysmans, W., Leys, C. \& Langenhove, H. V. Efficient toluene abatement in indoor air by a plasma catalytic hybrid system. Applied Catalysis B: Environmental 74, 161-169 (2007).

8.Zarra, T., Naddeo, V. \& Belgiorno, V. A novel tool for estimating the odour emissions of composting plants in air pollution management. Global Nest Journal 11(4),

9. Viccione, G., Zarra, T., Giuliani, S., Naddeo, V. \& Belgiorno, V. Performance Study of E-Nose Measurement Chamber for Environmental Odour Monitoring. Chemical Engineering 30,

10.Zarra, T., Giuliani, S., Naddeo, V. \& Belgiorno, V. Control of odour emission in wastewater treatment plants by direct and undirected measurement of odour emission capacity. Water Science and Technology 66, 1627-1633 (2012).

11.Zarra, T., Galang, M. G., Ballesteros, F., Belgiorno, V. \& Naddeo, V. Environmental odour management by artificial neural network - A review. Environment International 133, 105189 (2019).

12.Oliva, G. et al.. Next-generation of instrumental odour monitoring system (IOMS) for the gaseous emissions control in complex industrial plants. Chemosphere 271, 129768 (2021).

13.Naddeo, V., Zarra, T., Giuliani, S. \& Belgiorno, V. Odour Impact Assessment in Industrial Areas. Chemical Engineering 30,

14.Naddeo, V., Zarra, T., Oliva, G., Chiavola, A. \& Vivarelli, A. Environmental Odour Impact Assessment of Landfill Expansion Scenarios: Case Study of Borgo Montello (Italy). Chemical Engineering Transactions 54, 
15.Zarra, T., Reiser, M., Naddeo, V., Belgiorno, V. \& Kranert, M. A comparative and Critical Evaluation of Different Sampling Materials in the Measurement of Odour Concentration by Dynamic Olfactometry. Chemical Engineering Transactions 30,

16.Oliva, G. et al. Comparative evaluation of a biotrickling filter and a tubular photobioreactor for the continuous abatement of toluene. Journal of Hazardous Materials 380, 120860 (2019).

17.Oliva, G. et al.. Comparative analysis of AOPs and biological processes for the control of VOCs industrial emissions. Chemical Engineering Transactions 68, (2018).

18.Senatore, V., Zarra, T., Oliva, G., Belgiorno, V. \& Naddeo, V. Volatile organic compounds(VOCs) removal by combining bioscrubber and ozone pretreatment. Global Nest Journal 22,

19.Naddeo, V., Cesaro, A., Mantzavinos, D., Fatta-Kassinos, D. \& Belgiorno, V. Water and wastewater disinfection by ultrasound irradiation - a critical review. Issue 3 16, 561-577 (2014).

20.Zhang, Z., Du, X., Carlson, K. H., Robbins, C. A. \& Tong, T. Effective treatment of shale oil and gas produced water by membrane distillation coupled with precipitative softening and walnut shell filtration. Desalination 454, 82-90 (2019).

21.Senatore, V. et al.. Innovative membrane photobioreactor for sustainable CO2 capture and utilization. Chemosphere 273, 129682 (2021).

22.Pervez, M. N. et al. A critical review on nanomaterials membrane bioreactor (NMs-MBR) for wastewater treatment. npj Clean Water 3, (2020).

23.Millanar-Marfa, J. M. J. et al.. Self-forming Dynamic Membranes for Wastewater Treatment. Separation \& Purification Reviews 1-17 (2021) doi:10.1080/15422119.2021.1887223. 
Figure Captions

Figure 1. Emissioni Industriali 
Figures

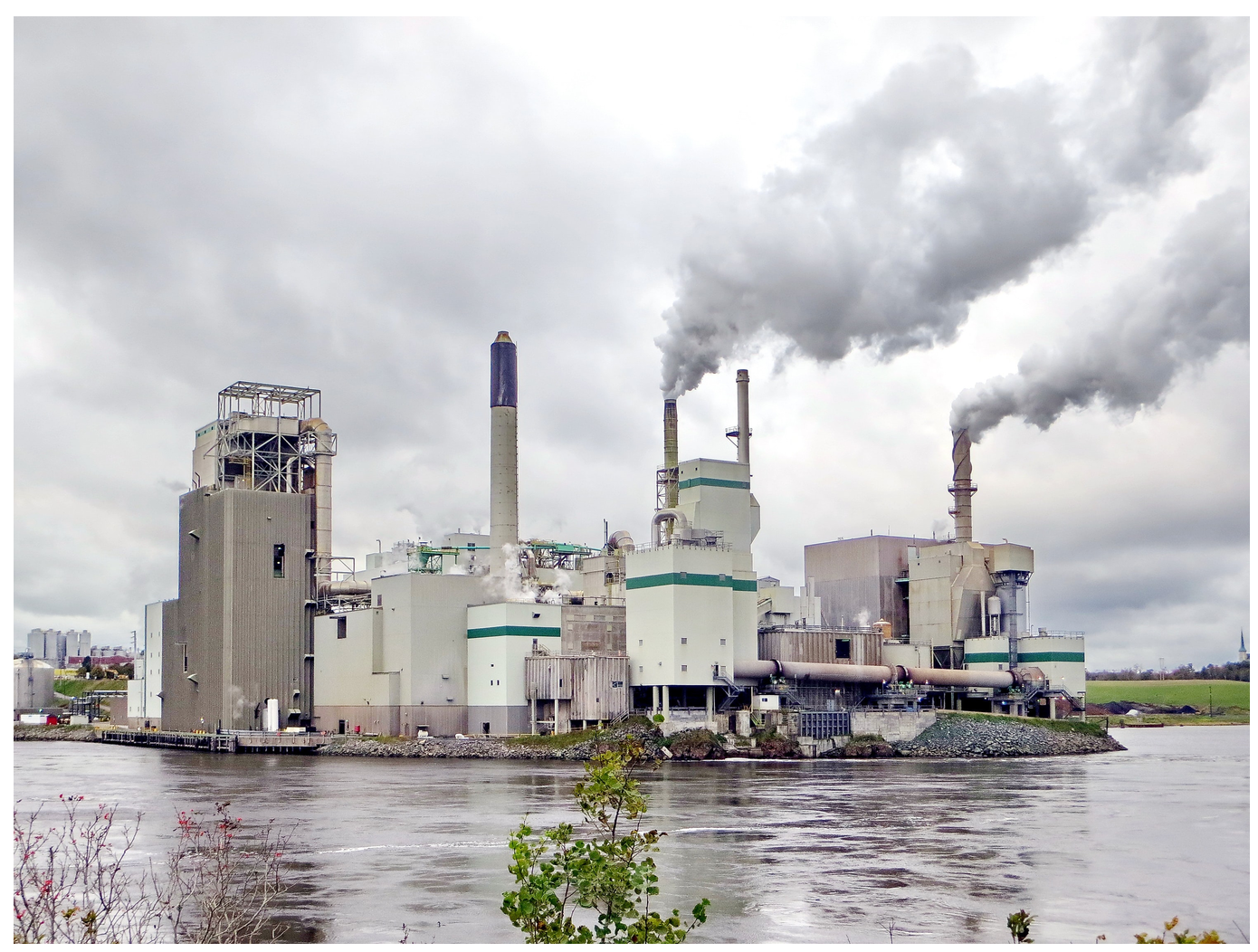

Figure 1: Emissioni Industriali 